\title{
XLVIII. STUDIES ON PECTIN.
}

\section{THE OXIDATION OF PECTIN BY FENTON'S REAGENT AND ITS BEARING ON THE GENESIS OF THE HEMICELLULOSES.}

\author{
BY ARTHUR GEOFFREY NORMAN \\ AND FREDERICK WALTER NORRIS. \\ From the Department of Biochemistry, University of Birmingham.
}

(Received March 3rd, 1930.)

IT is now some time since Nanji, Paton and Ling [1925] showed that the constitution of the unit of the pectin molecule might be expressed by a definite chemical formula. Their views seem to have met with fairly general acceptance, as evinced by a number of later publications by various authors. Little attempt, however, has been made subsequently to examine the consequences following the treatment of pectin as a definite chemical entity. A number of researches has been carried out on the condition and function of pectin in the plant tissues, while the metabolic changes undergone by the pectic substances during the ripening and senescence of fruits have received particular attention; but in most cases the physiological aspect has been predominant, to the neglect of the chemistry of the subject. This is unfortunate, since it is not possible to obtain a complete and true picture of the metabolism of any substance until the chemical changes which result in its formation and which occur in its degradation have been elucidated. Neither of these conditions is yet satisfied in the case of pectin, since there is no information as to its genesis, and little concerning its mode of breakdown and ultimate degradation products. The purpose of this investigation was to isolate certain degradation products of pectin by means not dissimilar to those possibly obtaining in the living plant.

Candlin and Schryver [1928] recognised the close generic relationship between pectin and the hemicelluloses, and proposed to include such substances under the general term polyuronides. They claimed to have converted pectin to hemicellulose by more or less prolonged treatment with caustic alkalis, and made the suggestion that the process of lignification involved the transformation of pectin to hemicellulose and subsequently, perhaps, to lignin by some synthetic process. Attempts by one of us to repeat the experiments of Candlin and Schryver have, up to the present, resulted only in failure; the alkali produced only much charred material at temperatures 
approaching $100^{\circ}$ and effected no appreciable change in the pectin at lower temperatures.

Nevertheless, the suggestion that pectin is converted to hemicellulose is an attractive one, based as it is on the fact that in non-lignified tissues cellulose and pectin are found, together with only small quantities of hemicellulose, whereas in lignified tissues we find cellulose, hemicelluloses and lignin, but only traces of pectin [O'Dwyer, 1925, 1926, 1928; Norris and Schryver, 1925].

The mechanism in the plant of the transformations indicated above is obscure, and few attempts have been made in the laboratory to imitate such changes. It would seem that the action of caustic alkalis is rather drastic, nor does it approximate to any action likely to take place in the living organism. A process of mild oxidation might, however, effect the conversion of pectin to an allied substance both in the plant and under laboratory conditions. Accordingly, attempts were made to treat pectin with suitable oxidising agents, and bromine in the presence of calcium carbonate was first employed, with indifferent success. The oxidation was extremely slow, and the products obtained were difficult to isolate owing to the presence of unchanged pectin. Later, the mild oxidation effected by Fenton's reagent-hydrogen peroxide in the presence of ferrous iron-suggested itself, and this proved to be the more useful reagent.

It will be recalled that the pectin formula of Nanji, Paton and Ling [1925] involves a unit consisting of one molecule of arabinose, one molecule of galactose and four molecules of galacturonic acid combined as anhydrides in a closed ring, the four carboxyl groups being free. From primary considerations it appeared possible that these carboxyl groups might be removed by the action of Fenton's reagent, and if at the same time the ring suffered no rupture then a product of the nature of a hexo-pentosan might be obtained.

As will appear in the experimental section, it was found that the action of Fenton's reagent was more vigorous than anticipated. The reaction was difficult to control; at temperatures below $30^{\circ}$ no action was evident, whilst above $35^{\circ}$ the pectin appeared to be oxidised completely away. Once commenced at $30^{\circ}$ the action was strongly exothermic, control of temperature was sometimes difficult and if the temperature rose above $35^{\circ}$ the yield of products precipitable by alcohol was considerably lowered.

Products in the form of white powders were obtained in each of a series of oxidations carried out on similar samples of a commercial pectin obtained from lemons and on calcium pectate similarly derived. These products are precipitable by alcohol and bear a strong physical resemblance to the hemicelluloses. Their almost complete lack of reducing power and their rather high rotatory power suggests that they are polymerised substances, and the results of analysis show that they are mixtures of substances whose simplest units consist of galactose and galacturonic acid combined in varying proportions. This result is a very surprising one and the original suggestion, that 
the acid groupings might be eliminated by Fenton's reagent, breaks down. The pectin ring appears to be broken, arabinose is lost and galactose and galacturonic acid remain. The reactions involved are probably much more complex than indicated by the foregoing, but at present no information is available which throws light on any intermediate stage of the completed reaction.

\section{ExPERIMENTAL.}

\section{Preparation of Fenton's reagent.}

The catalyst employed in this reaction is ferrous acetate, and since this is an unstable substance which readily oxidises in the air it is necessary to prepare it as required. About five parts by weight of barium acetate and three parts of ferrous sulphate are dissolved in a small volume of cold water. The filtered solution is employed in the reaction, but since the mixing has to be carried out in the cold, the precipitated barium sulphate is in such a fine condition that it passes good filter papers to a considerable extent. As will be seen later, the presence of barium sulphate tends to raise the ash content of the final product to a rather high figure, but this cannot easily be avoided. In some cases the barium sulphate was separated at the centrifuge, but this was not very satisfactory owing to partial oxidation of the ferrous acetate.

\section{Oxidation of pectin.}

The pectin employed in this work was a commercial product of good quality, with an ash content of $2 \cdot 2 \%$, prepared by the method of Wilson [1925]. One litre of a smooth solution of $2 \%$ concentration is placed in a large beaker and to this is added the iron catalyst as prepared by the method indicated above. For $20 \mathrm{~g}$. of pectin approximately $0 \cdot 2 \mathrm{~g}$. of ferrous iron is sufficient. The beaker is placed in a water-bath at $30^{\circ}$ and throughout the reaction the mixture is stirred as rapidly as possible with a strong stirrer provided with vanes. After the addition of iron, which causes the solution to stiffen and gel, 50 cc. of hydrogen peroxide ( 20 vols.) are run in at once, after which the viscosity of the solution rapidly diminishes. During the next 2 hours a further quantity of $150 \mathrm{cc}$. of hydrogen peroxide is added slowly from a dropping funnel and large quantities of carbon dioxide are evolved. Unless precautions are taken the temperature of the solution may rise to $50^{\circ}$ or more and this must on no account be permitted since it is found that the yield is much decreased if the temperature is allowed to exceed $35^{\circ}$. The surrounding water-bath has therefore to be cooled at this stage of the reaction, although unless the temperature of the solution is initially raised to about $30^{\circ}$ the reaction does not proceed to any extent. At the end of 4 hours the solution is filtered and poured into 2 to 4 volumes of alcohol, in which a white precipitate appears. This should be filtered off within a few hours, as the precipitate becomes greyish after longer contact with alcohol. A further small quantity of the product may be obtained by concentrating the alcoholic 
filtrate under diminished pressure and pouring into alcohol; this product is identical with the bulk of the precipitate. The total yield is small, about $5 \%$ on an average.

Since the Fenton reaction for the degradation of sugars of one series to those of a lower one is usually carried out on the calcium salt of the sugar acid, calcium pectate, in the form of a gel in aqueous suspension, was employed in some cases in lieu of the pectin solution. The calcium pectate gel is prepared by adding to a solution of pectin an equal volume of saturated lime water. After standing overnight, the resulting gel is thoroughly washed with water and suspended in water for the oxidation, which is carried out as previously described. By using the calcium gel as the starting point, the high viscosity which is given by the pectin solution in the early stages is avoided. However, there seemed to be no particular practical advantage in this, and further there was the grave disadvantage that even more inorganic material was introduced, with a resulting effect on the ash content of the final product.

Many samples were prepared under various conditions of time, temperature and concentration of pectin and peroxide, but the conditions indicated previously give the best yield of product, careful control of temperature being of major importance. If the reaction is unduly prolonged the oxidation proceeds to a stage at which no precipitate is given with alcohol. The precise nature of the products under these conditions has not been determined but it is believed that the pectin is completely oxidised to carbon dioxide and water.

The ash content of all preparations was high, due to the ash of the original pectin and the barium sulphate added with the catalyst. Various attempts were made to lower the ash content. Slight acidification of the dissolved product with hydrochloric acid, followed by dialysis, was unsuccessful since it was found that the products passed through good quality parchment, resembling in this respect certain water-soluble hemicelluloses. By repeated solution in slightly acidified warm water, filtration and reprecipitation the ash content was reduced. The amount of ash remaining, however, was even then quite appreciable; one sample, for instance, was dissolved and reprecipitated many times, but still retained nearly $8 \%$ of ash.

\section{Hydrolysis of the oxidation product.}

An examination of the products of hydrolysis was carried out in some detail. A small quantity of the preparation was boiled for several hours with $1 \%$ sulphuric acid and neutralised while still hot with barium carbonate. The precipitated barium sulphate and excess barium carbonate were filtered off and the filtrate divided into three portions.

(i) Phenylhydrazine was added and the osazone prepared in the usual manner. The purified osazone was obtained in the form of crystals which resembled those described for galactosazone, when examined microscopically. Recrystallised from $60 \%$ alcohol, the osazone had M.P. $181^{\circ}$; this is not in very close agreement with the recognised melting point of galactosazone- 
$191^{\circ}$-but the presence of small quantities of organic material may cause a lowering of the observed melting point.

(ii) Nitric acid was added to give a concentration of about $25 \%$ and the mixture gently heated on a water-bath for several hours. After standing overnight, considerable quantities of mucic acid were precipitated, indicating the presence of galactose or a galactose derivative.

(iii) To the third portion alcohol was added until a whitish precipitate appeared; this was filtered off and dissolved in $25 \%$ nitric acid. On treating this as in (ii) above, mucic acid was again precipitated. The white precipitate was therefore most probably the barium salt of galacturonic acid; this salt is soluble in water and would be formed when excess of barium carbonate was added to the hydrolysis liquid. Further critical examination was not possible owing to the small quantity of material available. Having regard to the nature of pectin it is impossible to conceive that the uronic acid present could be glycuronic acid; moreover, this acid yields a characteristic osazone, which was not detected. The presence of a uronic acid was amply confirmed by the results of quantitative analysis which will be discussed later. The two substances, galactose and galacturonic acid were the only ones detectable in the hydrolysis liquid. No trace of pentose was found, an observation which also was borne out by the results of analysis.

The progress of hydrolysis was followed under standardised conditions, samples being taken from the mixture at definite intervals and reducing sugars being determined by the iodimetric method of Baker and Hulton [1920]. The results in the following table are given in terms of the iodine equivalent to $100 \mathrm{~g}$. of the ash-free product, and recalculated to galactose by the use of the factor $0 \cdot 73$.

Table I. Rate of hydrolysis of the Fenton product.

$\begin{array}{ccc}\text { Time in hours } & \text { Iodine equivalent to } 100 \mathrm{~g} . & \text { Calculated as galactose } \\ 0 & \mathrm{~g} . & \mathrm{g} . \\ 0 \cdot 5 & 15 \cdot 17 & 10 \cdot 08 \\ 1 & 25 \cdot 67 & 18 \cdot 88 \\ 2 & 31 \cdot 50 & 23 \cdot 00 \\ 4 & 37 \cdot 30 & 27 \cdot 20 \\ 6 & 43 \cdot 01 & 31 \cdot 45 \\ 8 & 45 \cdot 90 & 33 \cdot 58 \\ 10 & 49 \cdot 40 & 36 \cdot 10 \\ & 51 \cdot 20 & 37 \cdot 40\end{array}$

It will be seen from Table I that the substance gives slight reduction before hydrolysis and that hydrolysis with sulphuric acid of the concentration employed $-1 \%$-is slow. The substance is therefore comparatively stable and the linkages within the molecule are strong.

The optical rotation of the samples was determined in some cases and the products were found to be strongly dextro-rotatory. Typical results were as follows:

$$
\begin{aligned}
& \text { Preparation (i) }[\alpha]_{D}^{20^{\circ}}=+162 \cdot 9^{\circ} \\
& \text { Preparation (ii) }[\alpha]_{D}^{20^{\circ}}=+161 \cdot 0^{\circ}
\end{aligned}
$$

Pectin itself has a specific rotation of the order of $+231^{\circ}$. 
Quantitative analysis. In addition to the determination of ash, estimations of total furfuraldehyde by the method of Tollens were carried out, and the yield of carbon dioxide obtained on hydrolysis with $12 \%$ hydrochloric acid was also determined by the method of Nanji, Paton and Ling [1925].

The content of uronic acid anhydride is obtained by multiplying the yield of carbon dioxide by 4 and is shown in the fourth column of Table II. It is also known that the uronic acid anhydrides yield approximately $16.66 \%$ of their weight of furfuraldehyde and consequently the theoretical amount of furfuraldehyde given by the uronic acid groups in the products can be calculated. This calculated figure appears in column 5 of Table II and it will be seen that within the limits of error this approximates to the actual yield of furfuraldehyde obtained experimentally. Hence it is clear that no pentose units are present and the conclusion previously arrived at, that galactose and galacturonic acid are the only products of hydrolysis, is justified.

\begin{tabular}{|c|c|c|c|c|c|}
\hline \multirow[b]{2}{*}{$\begin{array}{l}\text { Product } \\
\text { No. }\end{array}$} & \multirow[b]{2}{*}{$\begin{array}{c}\text { Ash } \\
\%\end{array}$} & \multicolumn{2}{|c|}{ Table II. } & \multicolumn{2}{|c|}{ Furfuraldehyde } \\
\hline & & $\begin{array}{c}\text { Carbon } \\
\text { dioxide } \\
\%\end{array}$ & $\begin{array}{c}\text { Uronic } \\
\text { anhydride } \\
\%\end{array}$ & $\begin{array}{c}\text { Calculated } \\
\%\end{array}$ & $\underset{\%}{\text { Found }}$ \\
\hline 1 & $17 \cdot 27$ & $15 \cdot 13$ & 60.52 & 10.08 & $10 \cdot 21$ \\
\hline 2 & $26 \cdot 65$ & 14.93 & $59 \cdot 72$ & 9.95 & 10.00 \\
\hline 3 & 26.55 & $\begin{array}{l}14 \cdot 44 \\
14.94\end{array}$ & 57.76 & $9 \cdot 61$ & 9.85 \\
\hline 4 & $7 \cdot 68$ & $13 \cdot 75$ & 55.00 & $9 \cdot 14$ & 8.65 \\
\hline 5 & $13 \cdot 38$ & 13.41 & $53 \cdot 64$ & 8.93 & $8 \cdot 84$ \\
\hline 6 & $14 \cdot 27$ & $13 \cdot 26$ & 53.04 & 8.83 & $8 \cdot 41$ \\
\hline 7 & $16 \cdot 42$ & 12.95 & 51.80 & $8 \cdot 63$ & 8.70 \\
\hline 8 & 30.98 & $12 \cdot 48$ & 49.92 & $8 \cdot 31$ & $8 \cdot 34$ \\
\hline
\end{tabular}

The table shows a series of products in which the amounts of galactose and galacturonic acid increase and decrease respectively as the table is descended. The last product approximates very closely to a simple unit corresponding to galactose-monogalacturonic acid (uronic acid anhydride content, $49 \cdot 4 \%$ ) and the first products approach the figure- $66.16 \%$ uronic acid anhydride-required by a substance whose simplest unit would be galactose-digalacturonic acid. It cannot be definitely stated, however, that these products represent mixtures of these two acids in polymerised form, since there might equally well be present acids whose simple units contain three or four galacturonic acid residues combined with galactose. Again, by an extension of the series in the opposite direction it may be possible to obtain mixtures containing such units as digalactose-monogalacturonic acid. Until further research has been carried out it is only possible to say that the products of oxidation of pectin are mixtures which yield on hydrolysis galactose and galacturonic acid in varying proportions, which depend on the exact conditions of the reaction. These mixtures probably represent stages in the reaction, and were the latter more susceptible to accurate control there seems no reason, in theory, why one or other of the constituents of the mixture should not solely be obtained, as, in fact, appears to be the case with sample No. 8. 


\section{Discussion.}

The oxidation of pectin by means of Fenton's reagent thus follows a wholly unexpected course. There is no production of the hexo-pentosan which theoretical considerations led us to expect. Nevertheless, the conversion of pectin to a mixture of substances with a strong generic resemblance to the hemicelluloses may be said to have been achieved. The two hemicelluloses of beechwood isolated by O'Dwyer [1926] yielded on hydrolysis arabinose and galacturonic acid, and xylose and glycuronic acid respectively. Norris and Preece [1930] have recently described hemicelluloses of wheat bran which yield in one case two sugars, and in other cases a sugar and a uronic acid on hydrolysis. Norman $[1929,1]$ has isolated hemicelluloses from cereal straws which yield, similarly, a uronic acid and pentose and hexose sugars. Schorger and Smith [1916] described a substance which they termed $\epsilon$-galactan, a water-soluble and slightly reducing product obtained from the wood of the Western Larch. Careful analysis by them showed that galactose was the only sugar present. The $\epsilon$-galactan, however, yielded $6 \cdot 18 \%$ of furfuraldehyde. It would seem now that this was undoubtedly due to galacturonic acid and that $\epsilon$-galactan is a substance not unlike those produced by us in the oxidation of pectin. The $6.18 \%$ of furfuraldehyde would be derived from $37.2 \%$ of galacturonic acid anhydride, the remaining $62.8 \%$ presumably consisting of anhydro-galactose. This corresponds very closely with the simple unit represented by digalactosegalacturonic acid. The conviction is therefore growing that the structural hemicelluloses will be found to exist universally as conjugated products, which yield on hydrolysis either two sugars, or, more generally a sugar or sugars, and a uronic acid.

Clearly then, such products as have been obtained by the mild oxidation of pectin fall within the category of hemicelluloses or polyuronides as indicated above. Meanwhile attempts are being made to separate the products obtained into their components.

Norman $[1929,2]$ has suggested that the formation of substances of the nature of pectin, hemicelluloses and the gums, can be accounted for by the mild oxidation of linked hexosan units. This theory therefore receives some support from the results described above. Oxidation by means of Fenton's reagent is being applied to natural products related to pectin with a view to the publication of further details of the method in the near future.

\section{SUMmaRY.}

1. Pectin may readily be oxidised by means of Fenton's reagent.

2. The reaction temperature must be carefully controlled, and must not be lower than $30^{\circ}$ nor higher than $35^{\circ}$.

3. The products of this exothermic reaction are mixtures, yielding on hydrolysis, galactose and galacturonic acid. They are possibly polymers containing mainly galactose-monogalacturonic acid and galactose-digalacturonic 
acid, although the presence of other conjugated acids containing galactose and galacturonic acid is not ruled out.

4. The products resemble in appearance and general properties the structural hemicelluloses, such as those obtained from wheat bran, beech wood and cereal straws.

5. Some support is lent to the view that the hemicelluloses may be formed in nature by the protracted mild oxidation of pectin.

\section{REFERENCES.}

Baker and Hulton (1920). Biochem. J. 14, 754.

Candlin and Schryver (1928). Proc. Roy. Soc. Lond. B 103, 365.

Nanji, Paton and Ling (1925). J. Soc. Chem. Ind. 44, $253 \mathrm{~T}$.

Norman $(1929,1)$. Biochem. J. 23, 1353.

$$
\text { (1929, 2). Biochem. J. 23, } 524 .
$$

Norris and Preece (1930). Biochem. J. 24, 59.

- and Schryver (1925). Biochem. J. 19, 676.

O'Dwyer (1925). Biochem. J. 19, 694.

- (1926). Biochem. J. 20, 656.

- (1928). Biochem. J. 22, 381.

Schorger and Smith (1916). J. Ind. Eng. Chem. 8, 494.

Wilson (1925). J. Ind. Eng. Chem. 17, 1065. 